\title{
Análise de rendimento cárneo das principais espécies de peixes comercializadas no Estado do Amazonas, Brasil
}

\author{
Antonio Fábio Lopes de SOUZA ${ }^{1}$, Antonio José INHAMUNS²
}

\begin{abstract}
RESUMO
A possibilidade do aproveitamento integral dos recursos pesqueiros tem produzido diversas pesquisas sobre os aspectos tecnológicos e nutricionais, objetivando estimular a exploraçāo comercial de várias espécies, bem como oferecer suporte para a implantação de indústrias na região. O presente trabalho determinou o rendimento cárneo de dez espécies com maior volume de desembarque no estado do Amazonas, visando fornecer dados mais recentes para a indústria de beneficiamento de pescado. As espécies foram selecionadas segundo dados estatísticos do IBAMA e coletadas em dois períodos sazonais (cheia e seca), no município de Manacapuru-AM. No período da cheia, os percentuais médios de rendimento das espécies atingiram 69,4\% de corpo limpo, 38,4\% de filé com pele e 30,7\% de filé sem pele, cortes preferenciais para comercialização. Na seca estes valores foram de $69 \% ; 37,6 \% ; 29,5 \%$, respectivamente. As dez espécies de peixes amazônicos estudadas apresentaram bom rendimento cárneo, com potencial para uso em diversos procedimentos tecnológicos.
\end{abstract}

PALAVRAS-CHAVE: Pescado de água doce, rendimento cárneo, variação sazonal.

\section{Meat yield analysis of the major fish species commercialized in the state of Amazonas, Brazil}

\section{ABSTRACT}

The possibility of whole use of fishery resources has led institutions and researchers to establish permanent studies on technological and nutritional aspects, aiming to stimulate commercial exploitation as well as to support the setting up industries in the region, that can make products of excellent quality based on regional fishes. This study determined the meat yield of ten species with the highest fish landing volume in Amazonas, aiming to provide more recent data for the fish processing industry. The species were selected according to data of IBAMA and collected in two seasons (flood and drought), in the municipality of Manacapuru-AM. In the flood period, the average yield reached $69.4 \%$ for gutted body, $38.4 \%$ for fillets with skin, and $30.7 \%$ for skinless fillets, which are the favored commercial cuts. In the drought period, data showed $69 \%$, $37.6 \%$, and $29.5 \%$ respectively. The analyses showed that the ten Amazon fish species have a high potential for use in several technological procedures.

KEYWORDS: Freshwater fish, meat yield, seasonal variation. 


\section{INTRODUÇÃO}

Há estimativas de que na Bacia Amazônica existam aproximadamente 3.000 espécies de peixes, o que representaria aproximadamente $30 \%$ da ictiofauna de água doce do mundo (Goulding 1980; Goulding et al. 1988). Mas, apesar da grande diversidade, poucas espécies são exploradas comercialmente para fins de alimentação da população regional (Falabella 1994; Batista et al. 1998).

A exploração e o consumo de muitas espécies regionais estáo limitados por alguns fatores como: o grande número de espinhas intramusculares em alguns grupos de peixes, a aparência bizarra de outros, a flacidez do tecido muscular, a falta de conhecimentos sobre a composição de nutrientes e, principalmente, ao "tabu alimentar" (Smith 1979; Falabella 1994; Evangelista 1998).

É evidente que o amazônida precisa diversificar as espécies para o consumo diário. Estudos realizados demonstraram que várias espécies de peixes amazônicos, com baixo valor comercial, apresentam um potencial nutricional que, em alguns casos, supera os peixes de maior aceitaçáo no mercado consumidor (Amorim e Inhamuns 2003; Braz Silva 2003; Gonzaga et al. 2005; Gonzaga e Inhamuns 2005).

A possibilidade do aproveitamento deste recurso tem gerado pesquisas permanentes sobre os aspectos tecnológicos e nutricionais de diversas espécies ícticas, objetivando estimular sua exploração comercial, bem como oferecer suporte para a implantação de indústrias na região, incentivando a fabricação de produtos à base de pescado regional de excelente qualidade. No entanto, apesar da constatação e divulgação do potencial que estas espécies apresentam em seus aspectos nutricionais e comerciais, continuam não fazendo parte do hábito alimentar da maioria da população regional que tem preferência pelas espécies consideradas nobres, uma realidade preocupante na região, que obriga pescadores e armadores a aumentarem o esforço de pesca sobre estas espécies.
A diversidade da ictiofauna amazônica possibilita a formação de muitos grupos de espécies, com formas anatômicas que influenciam diretamente no percentual quantitativo dos diversos cortes utilizados no processamento do pescado. O rendimento cárneo destas espécies depende da destreza do operário na retirada da parte cárnea, ou ainda, da eficiência das máquinas filetadoras e das características peculiares da matéria prima, como tamanho da cabeça, peso das vísceras, pele e nadadeiras.

Nesse contexto, a indústria de beneficiamento, que trata de espécies de baixo valor, empregando a tecnologia do pescado e agregando valores ao produto final, vem se erguendo sobre bases cada vez mais sólidas, gerando uma atividade lucrativa e benéfica para toda a populaçấo. Para subsidiar a atividade é necessário gerar dados recentes e seguros acerca do rendimento das principais espécies exploradas no Estado, e dessa forma, assegurar o planejamento e o crescimento do setor industrial do pescado. O presente trabalho foi um estudo descritivo com abordagem quantitativa na determinação do rendimento cárneo e resíduo gerado, das dez espécies de pescado com maior contribuiçáo no desembarque no Estado do Amazonas, em diferentes épocas do ano (cheia e seca).

\section{MATERIAL E MÉTODOS}

As principais espécies de peixes desembarcadas no estado do Amazonas (Tabela 1) foram definidas por meio de dados de desembarque do ano de 2004, provenientes de relatórios estatísticos do IBAMA (2005).

As amostras foram adquiridas no Terminal de Desembarque de Pescado no município de Manacapuru - AM (318'13"S e 60 $\left.37^{\prime} 11^{\prime \prime} \mathrm{W}\right)$. Foram coletados vinte exemplares de cada espécie, sendo então acondicionados em caixas isotérmicas com gelo em uma proporçáo de 1:1 (gelo:peixe).

Para o cálculo do rendimento cárneo, os exemplares foram medidos em ictiômetro e pesados em balança eletrônica digital,

Tabela 1- Lista de espécies amazônicas estudadas.

\begin{tabular}{llll}
\hline Ordem & Família & Nome Vulgar & Nome Científico \\
\hline \multirow{5}{*}{ Characiformes } & Prochilodontidae & Curimatã & Prochilodus nigricans (Agassiz, 1829) \\
\cline { 2 - 4 } & Prochilodontidae & Jaraqui de escama grossa & Semaprochilodus insignis (Schomburgk, 1841) \\
& Characidae & Matrinchã & Brycon amazonicus (Spix \& Agassiz, 1829) \\
& Characidae & Sardinha comprida & Triportheus auritus (Cuvier \&Valenciennes, 1850) \\
& Characidae & Pacu manteiga & Mylossoma duriventre (Cuvier, 1817) \\
\hline \multirow{3}{*}{ Siluriformes } & Characidae & Tambaqui & Colossoma macropomum (Cuvier, 1818) \\
& Pimelodidade & Mapará & Hypophythalmus edentatus (Spix \& Agassiz, 1829) \\
\hline Perciformes & Pimelodidade & Piramutaba & Brachyplatystoma vaillantii (Valenciennes, 1840) \\
\hline
\end{tabular}


marca Filizola ${ }^{\bullet}$ com sensibilidade de $2 \mathrm{~g}$, para a obtençáo do peso total e dos diferentes cortes; em seguida foram realizados os cálculos dos percentuais de rendimento em corpo limpo, filé com pele, filé sem pele (somente o músculo dorsal) e cabeça, segundo Morais et al. (1992), Contreras-Guzmán (1994) e Reidel et al. (2004):

$$
\eta=\frac{P f}{P i} \times 100
$$

Onde: $\eta=$ Rendimento

$P f=$ Peso final

$P i=$ Peso inicial

Os dados de rendimento dos lotes coletados nos períodos de cheia e seca foram comparados através da ANOVA de Fisher, que permitiu verificar em quais espécies a diferença de rendimento entre as duas épocas foi significante ao nível de 5\%. Utilizou-se o pacote estatístico STATISTICA, versão 8.0 (StatSoft 2007).

\section{RESULTADOS E DISCUSSÃO}

A análise dos resultados foi conduzida por meio da comparaçấo, entre período sazonal e características biométricas dos indivíduos amostrados, estabelecendo uma relação com o rendimento cárneo para cada espécie.

O número de amostras investigado por espécie e período sazonal, bem como os comprimentos e pesos médios estáo apresentados na Tabela 2.

As espécies estudadas apresentaram distribuição equitativa entre os períodos sazonais em relação a peso e comprimento.
Para os Characiformes curimatá, jaraqui, matrinchã e sardinha foram encontrados indivíduos maiores no período de cheia, enquanto que tambaqui e pacu foram maiores no período de seca. Para os Siluriformes encontraram-se maiores médias na cheia para piramutaba, e na seca para mapará e surubim. O tucunaré, representante dos Perciformes, foi o que apresentou maior tamanho médio quando capturado na seca. Alguns autores afirmam que o conhecimento da biometria pode ser útil como indicador do acúmulo de gordura e de desenvolvimento das gônadas, em função de diferenças ambientais e genéticas da espécie (Gurgel e Mendonça 2001; Silva Júnior et al. 2007). Estudos dessa natureza tem sido sugeridos porque, além de esclarecer aspectos referentes à biologia de uma determinada espécie, fornecem informaçóes valiosas sobre possíveis alteraçôes ambientais.

De dez espécies estudadas, sete não apresentaram diferenças significativas de rendimento nos diferentes cortes, no comparativo dos dois períodos de estudo (Tabela 3). Com exceção da espécie surubim que apresentou maior percentual de resíduos no período de seca, todos os demais cortes estudados alcançaram maiores valores no período de cheia.

Das dez espécies estudadas, os maiores valores de rendimento para corpo limpo e filé sem pele, os cortes preferencialmente comercializados, foram obtidos com o mapará (H. edentatus), nos dois períodos sazonais.

Lourenço et al. (1999) ao processarem mapará salgado seco encontraram rendimento cárneo total de $64,76 \%$, enquanto que Bicelli e Inhamuns (2002), ao processarem mapará para defumação a quente, determinaram para corpo limpo e filé sem pele valores de $85 \pm 1 \%$ e $45 \pm 2 \%$, respectivamente. Os menores percentuais de corpo limpo foram atribuídos ao tambaqui,

Tabela 2 - Médias de comprimento padrão $(\mathrm{cm})$ e peso $(\mathrm{g})$ das espécies de peixe com maior volume de desembarque, em dois períodos sazonais da bacia amazônica.

\begin{tabular}{lccccc}
\hline \multirow{2}{*}{ Espécie } & No & \multicolumn{2}{c}{ Cheia } & \multicolumn{2}{c}{ Seca } \\
\cline { 3 - 6 } & & C. padrão & Peso & C. padrão & Peso \\
\hline Curimatã & 20 & $29,8 \pm 3$ & $598,6 \pm 212$ & $22,1 \pm 4$ & $314,6 \pm 67$ \\
Jaraqui & 20 & $19,8 \pm 2$ & $215,7 \pm 49$ & $20,4 \pm 1$ & $178,2 \pm 51$ \\
Mapará & 20 & $31,4 \pm 6$ & $254,4 \pm 97$ & $33,6 \pm 4$ & $307,3 \pm 80$ \\
\hline Matrinchã & 20 & $29,3 \pm 1$ & $621,6 \pm 69$ & $24,5 \pm 5$ & $365,4 \pm 220$ \\
\hline Pacu & 20 & $17,3 \pm 1$ & $209 \pm 34$ & $18,8 \pm 1$ & $242 \pm 98$ \\
Piramutaba & 16 & $41,5 \pm 2$ & $1253,7 \pm 254$ & $38 \pm 2$ & $1108 \pm 354$ \\
\hline Sardinha & 20 & $20,9 \pm 2$ & $145,6 \pm 32$ & $18,3 \pm 1$ & $71,6 \pm 12$ \\
Surubim & 20 & $45 \pm 5$ & $1075 \pm 380$ & $48 \pm 6$ & $1536 \pm 480$ \\
\hline Tambaqui & 20 & $30,2 \pm 3$ & $1071,2 \pm 101$ & $32,1 \pm 2$ & $1229 \pm 53$ \\
\hline Tucunaré & 20 & $28,6 \pm 4$ & $589,8 \pm 97$ & $31 \pm 4$ & $699,8 \pm 201$ \\
\hline
\end{tabular}


Tabela 3 - Rendimento médio $(\alpha=0,05)$ em diferentes cortes nas dez espécies de peixes com maior volume desembarcado no Estado do Amazonas.

\begin{tabular}{|c|c|c|c|c|c|c|c|c|}
\hline \multirow{2}{*}{ Espécie } & \multirow{2}{*}{ Época } & \multicolumn{7}{|c|}{ Rendimento (\%) } \\
\hline & & Corpo limpo & Cabeça & Eviscerado & Filé com pele & Filé sem pele & Pele & Resíduos \\
\hline \multirow{5}{*}{ Curimatã } & Cheia & $70,53^{a}$ & $16,31^{\mathrm{a}}$ & $91,28^{a}$ & $40,19^{a}$ & $32,52^{\mathrm{a}}$ & $7,67^{\mathrm{a}}$ & $42,19^{a}$ \\
\hline & Seca & $65,55 b$ & $14,14 b$ & $79,70 \mathrm{~b}$ & $35,36 b$ & $29,54 b$ & $6,59 \mathrm{~b}$ & $46,74 b$ \\
\hline & $\mathrm{F}$ & 59,79 & 14,85 & 1163,86 & 21,66 & 11,33 & 5,71 & 17,14 \\
\hline & $p$ & $<0,0001$ & 0,0013 & $<0,0001$ & 0,0002 & 0,0037 & 0,0287 & 0,0007 \\
\hline & C.V. (\%) & 4,28 & 10,54 & 7,01 & 8,76 & 8,34 & 15,45 & 7,50 \\
\hline \multirow{5}{*}{ Jaraqui } & Cheia & $70,68^{a}$ & $14,10^{\mathrm{a}}$ & $84,01^{a}$ & $39,81^{a}$ & $30,91^{a}$ & $8,90^{\mathrm{a}}$ & $45,81^{a}$ \\
\hline & Seca & $69,54^{\mathrm{a}}$ & $14,86^{a}$ & $83,49^{a}$ & $41,86^{a}$ & $32,38^{a}$ & $9,49^{a}$ & $47,11^{a}$ \\
\hline & $\mathrm{F}$ & 0,62 & 0,02 & 0,10 & 0,16 & 0,59 & 0,39 & 1,13 \\
\hline & $\mathrm{p}$ & 0,4411 & 0,8769 & 0,7511 & 0,6909 & 0,4543 & 0,5385 & 0,3021 \\
\hline & C.V. (\%) & 4,44 & 19,81 & 4,08 & 13,73 & 16,25 & 14,19 & 13,82 \\
\hline \multirow{5}{*}{ Mapará } & Cheia & $79,40^{\mathrm{a}}$ & $12,50^{\mathrm{a}}$ & $91,56^{a}$ & $51,89^{a}$ & $39,00 \mathrm{a}$ & $12,89^{a}$ & $35,17^{a}$ \\
\hline & Seca & $74,88 \mathrm{~b}$ & $14,78 b$ & $90,01^{\mathrm{a}}$ & $48,29 b$ & $38,68^{a}$ & $9,61 b$ & $33,00 \mathrm{a}$ \\
\hline & $\mathrm{F}$ & 4,85 & 5,28 & 0,85 & 6,00 & 0,66 & 9,34 & 1,50 \\
\hline & $p$ & 0,0418 & 0,0346 & 0,3683 & 0,0255 & 0,4294 & 0,0071 & 0,2372 \\
\hline & C.V. (\%) & 6,38 & 17,07 & 4,92 & 8,01 & 10,08 & 24,40 & 9,97 \\
\hline \multirow{5}{*}{ Matrinchã } & Cheia & $69,81^{a}$ & $13,54^{a}$ & $83,18^{a}$ & $41,13^{a}$ & $31,66^{a}$ & $9,46^{\mathrm{a}}$ & $43,12^{\mathrm{a}}$ \\
\hline & Seca & $66,29^{a}$ & $15,80^{\mathrm{a}}$ & $88,20 \mathrm{~b}$ & $43,33 b$ & $30,50^{\mathrm{a}}$ & $12,83 b$ & $47,03^{a}$ \\
\hline & $\mathrm{F}$ & 2,00 & 0,80 & 14,84 & 5,95 & 2,73 & 6,49 & 0,39 \\
\hline & $p$ & 0,1765 & 0,3856 & 0,0014 & 0,0268 & 0,1181 & 0,0215 & 0,5417 \\
\hline & C.V. (\%) & 7,91 & 20,13 & 4,18 & 23,17 & 11,26 & 67,92 & 19,52 \\
\hline \multirow{5}{*}{ Pacu } & Cheia & $78,50^{\mathrm{a}}$ & $15,06^{a}$ & $93,85^{a}$ & $39,55^{a}$ & $30,65^{a}$ & $8,90^{\mathrm{a}}$ & $46,26^{a}$ \\
\hline & Seca & $75,63 b$ & $13,01 b$ & $87,67 \mathrm{~b}$ & $38,96^{a}$ & $29,38 b$ & $9,58^{a}$ & $50,72^{\mathrm{a}}$ \\
\hline & $\mathrm{F}$ & 8,42 & 17,12 & 63,41 & 4,09 & 6,87 & 0,25 & 3,21 \\
\hline & $p$ & 0,0099 & 0,0007 & $<0,0001$ & 0,0590 & 0,0179 & 0,6232 & 0,0912 \\
\hline & C.V. (\%) & 3,32 & 19,93 & 3,93 & 10,50 & 11,98 & 20,04 & 12,89 \\
\hline \multirow{5}{*}{ Piramutaba } & Cheia & $68,09^{a}$ & $16,92^{\mathrm{a}}$ & $84,99^{a}$ & $29,98^{a}$ & $24,92^{a}$ & $5,06^{a}$ & $45,76^{a}$ \\
\hline & Seca & $73,20 \mathrm{~b}$ & $11,06 \mathrm{~b}$ & $84,26^{a}$ & $29,54^{a}$ & $24,04^{\mathrm{a}}$ & $5,51 b$ & $56,89 \mathrm{~b}$ \\
\hline & $\mathrm{F}$ & 6,85 & 8,72 & 0,03 & 1,68 & 0,37 & 5,99 & 14,75 \\
\hline & $p$ & 0,0187 & 0,0094 & 0,8707 & 0,2128 & 0,5493 & 0,0263 & 0,0014 \\
\hline & C.V. (\%) & 12,02 & 33,32 & 10,69 & 13,52 & 14,09 & 19,87 & 16,96 \\
\hline \multirow{5}{*}{ Sardinha } & Cheia & $70,44^{a}$ & $17,94^{a}$ & $87,62^{a}$ & $42,21^{a}$ & $32,01^{a}$ & $10,20^{a}$ & $40,64^{a}$ \\
\hline & Seca & $67,35^{\mathrm{a}}$ & $19,80 \mathrm{~b}$ & $87,75^{a}$ & $38,64 b$ & $29,38 a$ & $9,26^{\mathrm{a}}$ & $41,06^{a}$ \\
\hline & $\mathrm{F}$ & 2,58 & 4,48 & 0,26 & 7,05 & 2,67 & 0,54 & 0,15 \\
\hline & $p$ & 0,1268 & 0,0494 & 0,6184 & 0,0167 & 0,1205 & 0,4721 & 0,7049 \\
\hline & C.V. (\%) & 5,49 & 10,48 & 3,87 & 7,91 & 11,18 & 23,05 & 5,72 \\
\hline \multirow{5}{*}{ Surubim } & Cheia & $65,55^{a}$ & $27,23^{a}$ & $92,59^{a}$ & $32,37^{a}$ & $28,01^{a}$ & $4,35^{a}$ & $40,53^{a}$ \\
\hline & Seca & $66,67^{a}$ & $12,74 b$ & $79,41 b$ & $32,60^{a}$ & $25,48^{a}$ & $7,12 b$ & $50,12 b$ \\
\hline & $\mathrm{F}$ & 0,14 & 279,01 & 14,89 & 0,02 & 3,31 & 6,96 & 18,18 \\
\hline & $p$ & 0,7140 & $<0,0001$ & 0,0012 & 0,9033 & 0,0854 & 0,0167 & 0,0005 \\
\hline & C.V. (\%) & 9,88 & 38,36 & 11,68 & 12,76 & 12,34 & 46,89 & 15,30 \\
\hline \multirow{5}{*}{ Tambaqui } & Cheia & $61,02^{\mathrm{a}}$ & $20,25^{a}$ & $82,34^{a}$ & $32,00 \mathrm{a}$ & $25,70^{a}$ & $6,29^{a}$ & $45,00 \mathrm{a}$ \\
\hline & Seca & $62,07^{a}$ & $22,44^{\mathrm{a}}$ & $84,51^{a}$ & $35,96 \mathrm{~b}$ & $29,08 b$ & $6,89^{a}$ & $37,63 b$ \\
\hline & $\mathrm{F}$ & 0,23 & 3,20 & 0,57 & 8,29 & 8,97 & 0,47 & 9,61 \\
\hline & $p$ & 0,6350 & 0,0903 & 0,4616 & 0,0100 & 0,0078 & 0,5026 & 0,0062 \\
\hline & C.V. (\%) & 7,76 & 13,55 & 7,64 & 10,66 & 10,95 & 28,98 & 15,50 \\
\hline \multirow{5}{*}{ Tucunaré } & Cheia & $61,02^{a}$ & $23,68^{a}$ & $84,51^{a}$ & $35,77^{a}$ & $31,84^{a}$ & $3,92^{\mathrm{a}}$ & $35,16^{a}$ \\
\hline & Seca & $62,92^{\mathrm{a}}$ & $25,29^{a}$ & $88,21 b$ & $37,96 \mathrm{~b}$ & $31,63^{a}$ & $6,33 b$ & $34,13 a$ \\
\hline & $\mathrm{F}$ & 2,78 & 2,48 & 7,60 & 6,87 & 0,06 & 26,55 & 1,59 \\
\hline & $p$ & 0,1125 & 0,1328 & 0,0130 & 0,0173 & 0,8142 & 0,0001 & 0,2229 \\
\hline & C.V. (\%) & 4,31 & 9,69 & 4,04 & 5,82 & 6,09 & 31,23 & 5,34 \\
\hline
\end{tabular}

Médias da mesma espécie seguidas por mesma letra nas colunas não diferiram entre si $(p>0,05)$; corpo limpo=sem cabeça, vísceras, nadadeiras e escamas; eviscerado=sem vísceras e guelras; resíduos = vísceras, ossos, nadadeiras, escamas e pele. 
nos dois períodos, e os de filé sem pele, para a piramutaba ( $B$. vaillantii), também nos dois períodos sazonais.

A porcentagem do corpo limpo de um pescado permite comparar as espécies, avaliar fatores críticos e visualizar o potencial para a industrializaçáo. O corpo limpo é caracterizado como um exemplar sem a cabeça, vísceras, nadadeiras e escamas. Carvalho (2003) encontrou percentual de $66,15 \%$ para o jaraqui (Semaprochilodus sp.), valor inferior ao encontrado nesta pesquisa em dois períodos sazonais; Oliveira e Inhamuns (2003) encontraram para o acará-prata índice de $68 \pm 3 \%$, bem acima do resultado desta pesquisa para o tucunaré, ambos da família Cichlidae e com o corpo comprimido lateralmente; e Amorim e Inhamuns (2002) determinaram para piracatinga (Callophysus macropterus), 77\% de corpo limpo, bem acima do encontrado neste estudo para o surubim, também pertencente a família Pimelodidae e com o corpo fusiforme deprimido.

As médias para todas as espécies, em filé sem pele, nos dois períodos ficaram abaixo da apresentada por ContrerasGuzmán (1994) para peixes de água doce (42,2\%). Porém, estão em conformidade aos encontrados por Castelo (1992), Carvalho (2003) e Oliveira e Inhamuns (2003) em estudos realizados com peixes amazônicos. Analisando o rendimento do filé sem pele do pirarucu (Arapaima gigas) criado em cativeiro, Oliveira (2007) encontrou percentual médio de $41,4 \pm 2,05 \%$, ligeiramente acima da média máxima determinada neste estudo para o mapará (39\%), e Barbosa et al. (2008) encontraram média de 33,19\% em duas linhagens de tilápia do Nilo (Oreochromis niloticus).

O rendimento do filé com pele variou entre 29,54 e 51,89 , percentuais apresentados pelas espécies piramutaba (B. vaillantii) e mapará (H. edentatus), respectivamente. Os valores encontrados estão consoantes com estudos realizados em jaraqui $(37,51)$, por Carvalho (2003) e por Faria et al. (2003) em tilápia do nilo (Oreochromis niloticus), com peso médio de $816 \mathrm{~g}(39,21)$. Entretanto, foram bem superiores aos valores encontrados por Simôes et al. (2007) em tilápia nilótica (Oreochromis niloticus) de origem tailandesa com 989,6 g, cujo percentual determinado foi de 21,63\% em filé com pele. Para Contreras-Guzmán (1994) o rendimento do filé com pele em pescado de água doce e marinho oscila entre $32,8 \%$ e 59,8\% com uma média geral de $50,5 \%$. Somente para peixes de água doce este percentual oscila em torno de $46,8 \%$.

O conhecimento do rendimento em pele é importante porque várias espécies são comercializadas sob a forma de filé com pele. Além disso, há um interesse crescente em aproveitála como matéria-prima de curtume, devido ao seu alto teor de colágeno. A pele perfaz em média 7,5\% do peso dos peixes ósseos (Contreras-Guzmán 1994). Neste estudo foram encontrados percentuais médios entre $3,92 \%$ e $12,89 \%$, no período de cheia, valores caracterizados na espécie tucunaré ( $C$. monoculus) e mapará (H. edentatus), respectivamente. A média geral deste corte entre as dez espécies estudadas foi 7,85\%.

O corpo limpo de pescado de água doce representa, em média, 63,73\% do peso do peixe (Contreras-Guzmán 1994), mas neste trabalho a média foi superior, com $69,36 \%$. Apenas o tambaqui (C. macropomum) e o tucunaré (C. monoculus) apresentaram valores mais baixos.

Souza et al. (2001) e Simóes et al. (2007) ao analisarem a mesma espécie de tilápia (Oreochromis niloticus) determinaram para o corpo limpo percentuais médios de 70,85\% e 76,89\%, respectivamente. Diferença semelhante no rendimento foi encontrada neste trabalho para Curimatã (70,53\% e 65,55\%) e piramutaba $(68,9 \%$ e $73,2 \%)$, quando comparadas em diferentes períodos sazonais.

A retirada apenas das vísceras de um pescado o classifica como eviscerado. O peixe eviscerado constitui-se em uma das formas de armazenamento e comercialização de pescados na Amazônia. Pescadores realizam esta prática, principalmente em espécies da família Pimelodidae, quando após a captura, os exemplares são eviscerados como forma de preservar as propriedades organolépticas e a qualidade. Dados referentes ao rendimento desta categoria de corte em pescados amazônicos ainda são escassos na literatura. Para este corte o maior valor foi obtido na espécie pacu ( $M$. duriventre), 93,85\%, na cheia e o menor no curimatã (P. nigricans), com 79,7\%, na seca.

Os resíduos dos exemplares estudados foram divididos em duas classes: cabeça e demais resíduos (vísceras, escamas, nadadeiras e ossos). A determinação da contribuição da cabeça apresentou valores médios em todo período de estudo entre $11 \%$ e $27 \%$, sendo que o maior percentual foi obtido com a espécie surubim (P. fasciatum) com $27,23 \%$ na cheia, e o menor com a piramutaba (B. vaillantii) com $11,06 \%$ no período de seca. Em pescado, o tamanho da cabeça é inversamente proporcional ao rendimento potencial (Morais et al. 1992). Neste estudo, as espécies que apresentaram maior percentual de cabeça durante todo o ano, tucunaré e tambaqui, também foram as que apresentaram menor rendimento em corpo limpo.

Castelo (1992) encontrou valores médios de cabeça na faixa de 18,95\% em espécies de peixes amazônicos e Contreras-Guzmán (1994) relata percentuais médios de $17,76 \%$ para peixes de água doce. Para os demais resíduos (vísceras, escamas, nadadeiras e ossos) a variação em todo período de estudo oscilou entre $33 \%$ e $56,89 \%$, valores estes atribuídos às espécies mapará (H. edentatus) e piramutaba ( $B$. vaillantii), respectivamente. Na cheia o maior percentual foi para o pacu e na seca para a piramutaba. Os dados obtidos nesta pesquisa estão próximos aos encontrados por Castelo (1992), que determinou percentuais médios para resíduos em espécies amazônicas na ordem de 42,24\%. 


\section{CONCLUSÕES}

A espécie mapará foi a que apresentou o maior percentual para o corte "corpo limpo" na cheia, enquanto que filé com pele, filé sem pele e pele foram superiores tanto na seca quanto na cheia. Constatou-se que o mapará, independente da época do ano, é o pescado mais indicado para processamento nos cortes preferenciais para comercialização.

Entre as espécies estudadas, o pacu apresentou o maior percentual para corpo limpo no período de seca e no corte eviscerado na cheia, caracterizando a espécie como de bom rendimento para consumo nos dois períodos de estudo.

O surubim, na cheia, apresentou o maior valor para o corte "cabeça" e na seca, maior valor para "resíduos". A espécie tucunaré, na seca, apresentou maior percentual para o corte "cabeça". O pacu apresentou maior percentual de resíduos na cheia. Os valores apresentados indicaram estes como sendo os mais apropriados para o aproveitamento deste corte na elaboração de subprodutos, como ração animal, a partir de resíduos de pescados.

O jaraqui, a sardinha e o curimatá, espécies que alcançam baixos valores em época de safra, apresentaram bom rendimento, se comparados a outras espécies anteriormente estudadas.

\section{BIBLIOGRAFIA CITADA}

Amorim, R.M.S.; Inhamuns, A.J. 2003. Products made from species with low commercial value. In: XIII Congresso Brasileiro de Engenharia de Pesca, Bahia. Porto Seguro: Associação dos Engenheiros de Pesca da Bahia. V. Único. (in Portuguese).

Amorim, R.M.; Inhamuns, A.J. 2002. Physico-chemical and technological application of small catfish caught in Amazonas State. In: XI Congresso de Iniciação Cientifica da UFAM, Universidade Federal do Amazonas, Manaus - AM. (in Portuguese).

Barbosa, A.C.B.; Carneiro, P.L.S.; Malhado, C.H.M.; Affonso, P.R.A.M.; Carneiro, J.C.S.C.; Rocha, L.G.; Carneiro, J.D.S. 2008. Performance and Sensorial Evaluation in Nile Tilapia (Oreochromis niloticus). Revista Científica de Produção Animal, 10 (1): 50-59. (in Portuguese, with abstract in English).

Batista, V.S.; Inhamuns, A.J.; Freitas, C.E.C.; Freire-Brasil, D. 1998. Characterization of the fishery in riverine communities in the Low-Solimões/High-Amazon region. Fisheries Management and Ecology, 5: 101-117.

Bicelli, B.C.; Inhamuns, A.J. 2002. Physicochemical and sensory characterization of mapará (Hypophthalmus spp.) smoked. In: XI Congresso de Iniciação Científica da UFAM, Universidade Federal do Amazonas, Manaus - AM. (in Portuguese).

Braz Silva, M. 2003. Using piranha Serrassalmus spp. to elaborate fish soup. Monografia de Graduação, Universidade Federal do Amazonas, Manaus, Amazonas. 36 pp. (in Portuguese).
Carvalho, N.L.A. 2003. Effects of physical and chemical factors on the formation of gels in "surimi" of two commercial fish species in the Amazon. Tese de Doutorado, Instituto Nacional de Pesquisas na Amazônia/Universidade Federal do Amazonas, Manaus, Amazonas. 145 pp. (in Portuguese).

Castelo, F.P. 1992. Rational use of freshwater fish of the Amazon. Evaluation of freshness of Jaraqui (Semaprochilodus taeniurus e Semaprochilodus insignis). Acta Amazonica, 22 (3): 437-448. (in Portuguese, with abstract in English).

Contreras-Guzmán, E.C. 1994. Biochemistry of fishery products. FUNEP, Jaboticabal, SP. 409 pp. (in Portuguese)

Evangelista, J.P. 1998. Food Technology. 2a Ed. Atheneu, SP. p. 313315. (in Portuguese).

Falabella, P.G. 1994. Fishing in the Amazon River: Problems and Solutions. Edua, Manaus, Amazonas. 156 pp. (in Portuguese).

Faria, R.H.S.; Souza, M.L.R.; Wagner, P.M.; Povh, J.A.; Ribeiro, R.P. 2003. Processing yield of Nile tilapia (Oreochromis niloticus Linnaeus, 1757) and pacu (Piaractus mesopotamicus Holmberg, 1887). Acta Scientiarum. Animal Sciences, 25 (1): 21-24. (in Portuguese, with abstract in English).

Gonzaga, L.S.; Inhamuns, A.J. 2005. Formulation of nuggets from fish with low commercial value of the Amazonas State. In: XII Congresso Brasileiro de Engenharia de Pesca, Ceará. v. Único. Fortaleza: Associaçáo dos Engenheiros de Pesca do Ceará, Fortaleza, Ceará. v. Único. (in Portuguese).

Gonzaga JR., M.A.; Carvalho, N.L. de A.; Carvalho, M.A.F. 2005. Production of restructured fish with higher added value made from fish species in the Amazon region. In: XII Congresso Brasileiro de Engenharia de Pesca. v. Único. Fortaleza: Associação dos Engenheiros de Pesca do Ceará, Fortaleza, Ceará. (in Portuguese).

Goulding, M. 1980. The fishes and the forest: explorations in Amazonian natural history. University of California Press, Berkeley, CA, USA. 280 pp.

Goulding, M.; Carvalho, M.L.; Ferreira, E.G. 1988. Rio Negro: rich life in poor water: Amazonian diversity and foodplain ecology as seen through fish communities. The Hague: SPB Academic Publishing. 200 pp.

Gurgel, H.C.B.; Mendonça, V.A. 2001. Population structure of Astyanax bimaculatus vittatus (Castelnau, 1855) (Characidae, Tetragonopterinae) from Ceará Mirim River, Poço Branco, RN. Revista Ceres, Viçosa, 48 (276): 159-168. (in Portuguese, with abstract in English).

Ibama. Instituto Brasileiro do Meio ambiente e dos Recursos Naturais Renováveis. 2005. Fisheries Statistics 2004, Brazil Large regions and units of the federation. IBAMA, Brasília. 98 pp. (in Portuguese).

Lourenço, L.F.H.; Fernandes, G.M.L.; Cintra, I.H.A. 1999. Physical, chemical and microbiological features marará Hypophthalmus edentatus Spix, 1829 salted and dried in solar, p. 314-321. In: Anais do XI CONBEP e do I CONLAEP v.1. Recife. (in Portuguese). 
Morais, C.; Mantovani, D.M.B.; Carvalho, C.R.L. 1992. Yield and chemical composition of flesh ictiofauna to catch shrimpseven-beards (Xiphopenaeus kroyeri, Heller, 1982). Coletâneas do ITAL, Campinas, 22(1): 62-72. (in Portuguese, with abstract in English).

Oliveira, P.R. 2007. Quality of the pirarucu (Arapaima gigas Schinz 1822) coming from pisciculture, stored in ice, frozen and its derivatives. Tese de Doutorado, Instituto Nacional de Pesquisas da Amazônia/Universidade Federal do Amazonas, Manaus, Amazonas. 108 pp. (in Portuguese).

Oliveira, M.J.M.; Inhamuns, A.J. 2003. Technological characterization of Discus silver (Chaetobranchus semifasciatus) originating in the Amazon Basin. In: XII Congresso de Iniciação Cientifica - CNPq/UFAM, Universidade Federal do Amazonas, Manaus - AM. (in Portuguese).

Reidel, A.; Oliveira, L.G.; Piana, P.A.; Lemainski, D.; Bombardelli, R.A.; Boscolo, W.R. 2004. Evaluation of yield and morphometric characteristics of curimbatá Prochilodus lineatus (Valenciennes, 1836), and piavuçu Leporinus macrocephalus (Garavello \& Britski, 1988) males and females. Varia Scientia, 4 (8): 71-78. (in Portuguese, with abstract in English).
Silva Júnior, M. G.; Castro, A. C. L.; Soares, L. S.; França, V. L. 2007. Length-weight relationship of fish species from the estuary Patience River of Maranhão Island, Brazil. Boletim do Laboratório de Hidrobiologia, 20: 30-37. (in Portuguese, with abstract in English).

Simões, M.R.; Ribeiro, C.F.A.; Ribeiro, S.C.A.; Park, K.J.; Murr, F.F.E.X. 2007. Physicochemical and microbiological composition and yield of thai-style tilapia fillets (Oreochromis niloticus). Ciência e Tecnologia de Alimentos, 27(3): 608-613. (in Portuguese, with abstract in English).

Smith, N.J.H. 1979. Fishing in the Amazon River. INPA/CNPq. 154 pp. (in Portuguese).

Souza, M.L.R.; Maranhão, T.C.F. 2001. Carcass, fillet and byproducts yield of filleting of Nile tilapia Oreochromis niloticus (L.) in relation to body weight. Acta Scientiarum 23 (4): 897-901. (in Portuguese, with abstract in English).

StatSoft, Inc. 2007. STATISTICA (data analysis software system), version 8.0. www.statsoft.com.

Recebido em 24/03/2010

Aceito em 05/07/2010 
